\title{
Modulated crystal structure of the atypical charge density wave state of single-crystal $\mathrm{Lu}_{2} \mathrm{Ir}_{3} \mathrm{Si}_{5}$
}

\author{
Sitaram Ramakrishnan $\odot,{ }^{1, *}$ Andreas Schönleber $\odot,{ }^{1}$ Jin-Ke Bao $\odot,{ }^{1}$ Toms Rekis $\odot,{ }^{1}$ Surya Rohith Kotla, ${ }^{1}$ \\ Achim M. Schaller $\odot,{ }^{1}$ Sander van Smaalen $\odot,{ }^{1, \dagger}$ Leila Noohinejad, ${ }^{2}$ Martin Tolkiehn, ${ }^{2}$ Carsten Paulmann, ${ }^{3}$ \\ N. S. Sangeetha $\odot,{ }^{4, \$}$ Dilip Pal $\odot,{ }^{4}$ Arumugam Thamizhavel, ${ }^{5}$ and Srinivasan Ramakrishnan $\odot^{5,8}$ \\ ${ }^{1}$ Laboratory of Crystallography, University of Bayreuth, 95447 Bayreuth, Germany \\ ${ }_{2}^{2}$ P24, PETRA III, DESY, 22607 Hamburg, Germany \\ ${ }^{3}$ Mineralogisch-Petrographisches Institut, Universität Hamburg, 20146 Hamburg, Germany \\ ${ }^{4}$ Department of Physics, Indian Institute of Technology, Guwahati 781039, India \\ ${ }^{5}$ Department of Condensed Matter Physics and Materials Science, Tata Institute of Fundamental Research, Mumbai 400005, India
}

(Received 25 March 2021; revised 29 July 2021; accepted 5 August 2021; published 24 August 2021)

\begin{abstract}
The three-dimensional charge density wave (CDW) compound $\mathrm{Lu}_{2} \mathrm{Ir}_{3} \mathrm{Si}_{5}$ undergoes a first-order CDW phase transition at around $200 \mathrm{~K}$. An atypical CDW state is found, that is characterized by an incommensurate CDW with $\mathbf{q}=[0.2499(3), 0.4843(4), 0.2386(2)]$ at $60 \mathrm{~K}$, and a large orthorhombic-to-triclinic lattice distortion with $\beta=91.945(2)^{\circ}$. We present the modulated crystal structure of the incommensurate CDW state. Structural analysis shows that the CDW resides on the zigzag chains of iridium atoms along $\mathbf{c}$. The structural distortions are completely similar between nonmagnetic $\mathrm{Lu}_{2} \mathrm{Ir}_{3} \mathrm{Si}_{5}$ and previously studied isostructural magnetic $\mathrm{Er}_{2} \mathrm{Ir}_{3} \mathrm{Si}_{5}$ with the small differences explained by the different values of the atomic radii of Lu and Er. Such a similarity is unique to $R_{2} \mathrm{Ir}_{3} \mathrm{Si}_{5}$ ( $R=$ rare earth). It differs from, for example, the rare-earth CDW compounds $R_{5} \mathrm{Ir}_{4} \mathrm{Si}_{10}$ for which $\mathrm{Lu}_{5} \mathrm{Ir}_{4} \mathrm{Si}_{10}$ and $\mathrm{Er}_{5} \mathrm{Ir}_{4} \mathrm{Si}_{10}$ possess entirely different CDW states. We argue that the mechanism of CDW formation, thus, is different for $R_{2} \mathrm{Ir}_{3} \mathrm{Si}_{5}$ and $R_{5} \mathrm{Ir}_{4} \mathrm{Si}_{10}$.
\end{abstract}

DOI: 10.1103/PhysRevB.104.054116

\section{INTRODUCTION}

Coexistence and competition of multiple phase transitions observed in both inorganic and organic compounds have always attracted the attention of theorists and experimentalists [1-4]. For instance, charge density wave (CDW) ordering has been found to coexist with superconductivity or magnetism in a variety of rare-earth intermetallic compounds [5]. An important feature of the observed CDWs in those compounds is the absence of explicit low dimensionality in their crystal structures. It is worthwhile to recall here, that originally CDWs are expected to occur in materials which possess quasione-dimensional (1D) structures [6]. It was suggested that such 1D structures favor nesting of the Fermi surface that leads to the CDW transition. However, CDWs have been discovered in a large number of compounds which do not belong to this category. Examples include $\alpha$-uranium [7], $\mathrm{CuV}_{2} \mathrm{~S}_{4}$ [8-11], $\mathrm{La}_{3} \mathrm{Co}_{4} \mathrm{Sn}_{13}$ [12-14], $R \mathrm{Te}_{3}(R=\mathrm{La}, \mathrm{Sm}, \mathrm{Gd}, \mathrm{Tb}, \mathrm{Dy}$, $\mathrm{Ho}$, Er, and Tm) [15-17], $R \mathrm{Te}_{2}(R=\mathrm{La}$ and Ce) $[18,19]$, $R_{5} \mathrm{Ir}_{4} \mathrm{Si}_{10}\left(R=\mathrm{Dy}, \mathrm{Ho}, \mathrm{Er}, \mathrm{Yb}\right.$, and $\mathrm{Lu}$ ) [5], and $\mathrm{Sm}_{2} \mathrm{Ru}_{3} \mathrm{Ge}_{5}$ [20]. Temperature-dependent single-crystal $\mathrm{x}$-ray diffraction (SXRD) experiments have established multiple phase transitions with incommensurate and commensurate CDWs in

\footnotetext{
*Present address: Department of Quantum Matter, AdSM, Hiroshima University, Higashi-Hiroshima 739-8530, Japan.

†smash@uni-bayreuth.de

†Present address: Institute for Quantum Materials and Technologies, Karlsruhe Institute of Technology, 76021 Karlsruhe, Germany.

§ramky@tifr.res.in
}

several compounds $R \mathrm{NiC}_{2}(R=\mathrm{Ce}, \mathrm{Pr}, \mathrm{Nd}, \mathrm{Sm}, \mathrm{Gd}, \mathrm{Tb}$, Dy, $\mathrm{Ho}, \mathrm{Er}$, and Tm) [2,21-26]. The CDW in $\mathrm{SmNiC}_{2}$ is completely suppressed upon entering the ferromagnetic (FM) state $[21,22]$. On the other hand, Kolincio et al. [26] have recently discovered coexistence of CDW order and field-induced FM order in $\mathrm{TmNiC}_{2}$.

$\mathrm{Lu}_{2} \mathrm{Ir}_{3} \mathrm{Si}_{5}$ has a complex three-dimensional orthorhombic crystal structure with space-group Ibam at $300 \mathrm{~K}$. Earlier studies on polycrystalline $\mathrm{Lu}_{2} \mathrm{Ir}_{3} \mathrm{Si}_{5}$ suggested the presence of a CDW phase transition at around $200 \mathrm{~K}$ [27,28]. Subsequent transmission electron microscope (TEM) measurements established an incommensurate modulation below $200 \mathrm{~K}$ with modulation wave-vector $\mathbf{q}=\delta(\overline{1} 21)$ and $\delta=0.23-0.25$ [29], thus, confirming the CDW character. Investigations on single-crystalline material revealed anomalies in the resistivity $(\rho)$, heat-capacity $\left(C_{p}\right)$, and magnetic susceptibility $(\chi)$, suggesting a strongly coupled (electron-phonon) firstorder CDW transition at around $200 \mathrm{~K}$ [30]. $\mathrm{Lu}_{2} \mathrm{Ir}_{3} \mathrm{Si}_{5}$ has also been shown to exhibit superconductivity below $3.5 \mathrm{~K}$ [27].

Previously, we have investigated the CDW of the isostructural compound $\mathrm{Er}_{2} \mathrm{Ir}_{3} \mathrm{Si}_{5}$ [31-33]. SXRD has revealed superlattice reflections below $150 \mathrm{~K}$ at similar positions as found for $\mathrm{Lu}_{2} \mathrm{Ir}_{3} \mathrm{Si}_{5}$, that is, $\mathbf{q}=[0.2495(2), 0.4973(1), 0.2483(1)]$ at $75 \mathrm{~K}$. However, we have discovered a strong monoclinic lattice distortion, which develops at the CDW phase transition [33]. We found that the actual symmetry of the lowtemperature crystal structure of the CDW phase is triclinic. The lattice distortion and concomitant lowering of point symmetry might actually be the driving force for development of the CDW state. Recently, $\mathrm{BaFe}_{2} \mathrm{Al}_{9}$ was also reported to 
develop an incommensurate CDW accompanied by a strong lattice distortion [34].

Here we report the low-temperature crystal structure of $\mathrm{Lu}_{2} \mathrm{Ir}_{3} \mathrm{Si}_{5}$ in its CDW state. We find that the incommensurately modulated structure is similar to that of $\mathrm{Er}_{2} \mathrm{Ir}_{3} \mathrm{Si}_{5}$ in its CDW state [33]. We discuss the similarities and subtle differences between the two compounds.

\section{EXPERIMENT}

Single crystals of $\mathrm{Lu}_{2} \mathrm{Ir}_{3} \mathrm{Si}_{5}$ were grown in a tetra-arc furnace from a stoichiometric mixture of the elements, employing a modified Czochralski technique. The purity of the reactants was $99.99 \%$ for $\mathrm{Lu}$ and Ir and $99.999 \%$ for silicon. The 2:3:5 stoichiometry was established by energy-dispersive $\mathrm{X}$-ray analysis. X-ray diffraction confirmed the expected lattice parameters. The crystal was then used to study the physical properties reported in Ref. [30]. From the same crystal a small part was crushed, and a piece of $0.13 \times 0.09 \times$ $0.06 \mathrm{~mm}^{3}$ was selected for the present SXRD experiments.

$\mathrm{X}$-ray diffraction experiments were performed at Beamline P24 of PETRA-III extension at DESY in Hamburg, employing radiation of a wavelength of $0.5000 \AA$. The temperature of the specimen was controlled by a CRYOCOOL open-flow helium gas cryostat. Complete data sets of intensities of Bragg reflections were measured at temperatures of 298, 260, and $230 \mathrm{~K}$ [orthorhombic room-temperature (RT) phase], $200 \mathrm{~K}$ (mixed phase), and 120, 60, and $20 \mathrm{~K}$ (CDW phase). Experimental details are listed in Sec. S1 of the Supplemental Material [35].

In a second approach, the SXRD data were used to produce undistorted sections of reciprocal space, employing the software CRYSALISPRO [36]. The $(h 0 l)$ sections clearly show the orthorhombic lattice at $298 \mathrm{~K}$ [Fig. 1(a)], split reflections of the two domains of the pseudomonoclinic lattice at $60 \mathrm{~K}$ [Fig. 1(c)], and the simultaneous presence of orthorhombic and pseudomonoclinic reflections at $200 \mathrm{~K}$ [Fig. 1(b)].

\section{RESULTS AND DISCUSSION}

Upon cooling the crystal, the SXRD experiments confirmed the Ibam crystal structure of $\mathrm{Lu}_{2} \mathrm{Ir}_{3} \mathrm{Si}_{5}$ at 298, 260, and $230 \mathrm{~K}$. At $200 \mathrm{~K}$ we observe the coexistence of two phases: the orthorhombic RT phase and the low-temperature CDW phase (Fig. 1). The latter is characterized by superlattice reflections at positions $\mathbf{q}=[0.2523(7), 0.4685(7), 0.2254(4)]-$ in agreement with TEM results [29] —and a strong monoclinic lattice distortion with $\beta=92.027(3)^{\circ}$ (Fig. 2; also see Fig. S1 and Table S4 in the Supplemental Material [35]). The coexistence of the two phases at $200 \mathrm{~K}$ as well as the discontinuities in the lattice parameters are in agreement with the first-order character of the CDW transition.

At 120,60 , and $20 \mathrm{~K}$ only the $\mathrm{CDW}$ phase remains. Cooling from 200 to $20 \mathrm{~K}$ results in a tendency of the modulation wave vector towards the commensurate value $(1 / 4,1 / 2,1 / 4)$, but it never reaches this value with $\mathbf{q}=[0.2494(3), 0.4848(4), 0.2378(2)]$ at $120 \mathrm{~K}$ and $\mathbf{q}=$ $[0.2499(2), 0.4868(4), 0.2388(2)]$ at $20 \mathrm{~K}$ (see Table S3 in the Supplemental Material [35]).

The obvious lattice distortion implies monoclinic or lower symmetries for the CDW phase (Fig. 2). However, consid-

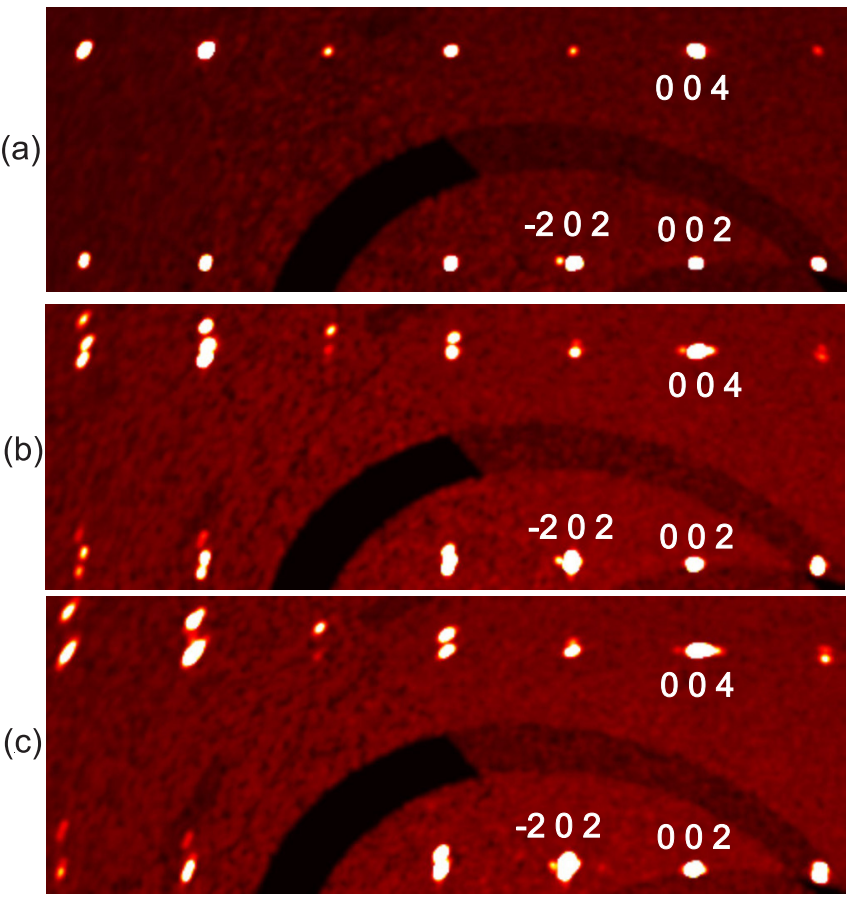

FIG. 1. ( $h 0 l$ ) section of reciprocal space, reconstructed from the SXRD data measured at: (a) 298, (b) 200, and (c) 60 K. Splitting of reflections due to the pseudomonoclinic lattice distortion increases with an increasing magnitude of the index $h$. The black bands are due to bands of insensitive pixels between individual modules of the Pilatus $1 \mathrm{M}$ CdTe detector.

eration of the point symmetry of the diffraction leads to the conclusion of triclinic symmetry with superspace group $I \overline{1}(\alpha \beta \gamma) 0$ (No. 2.1.1.1 with standard setting $P \overline{1}(\alpha \beta \gamma) 0$ [37]). See Sec. S3 of the Supplemental Material [35]. This is the same symmetry as has been found for the CDW phase of $\mathrm{Er}_{2} \mathrm{Ir}_{3} \mathrm{Si}_{5}$ [33].

Lowering of the point symmetry at a phase transition opens up the possibility of formation of a twinned crystal. The triclinic lattice can form out of the orthorhombic lattice in four different but equivalent orientations (Fig. 3). This implies four possible twin domains. The SXRD data have been analyzed under consideration of these four domains (see the discussion in Sec. S2 of the Supplemental Material [35]). One result is that our crystal formed with unequal volume fractions of the domains in the CDW phase. Furthermore, we have observed that the crystal quality deteriorates upon cooling through the phase transition, even more so for $\mathrm{Lu}_{2} \mathrm{Ir}_{3} \mathrm{Si}_{5}$ than previously found for $\mathrm{Er}_{2} \mathrm{Ir}_{3} \mathrm{Si}_{5}$. This is probably caused by the strain induced by the lattice distortions. The combination of broadened Bragg reflections with twinning then results in many partially overlapping Bragg reflections that could not be used for structural analysis.

At $60 \mathrm{~K}$, the superlattice reflections of one domain can be indexed by the incommensurate modulation wave-vector,

$$
\mathbf{q}^{1}=[0.2499(2), 0.4843(4), 0.2386(2)]
$$

The modulation wave vector is similar to those observed by electron diffraction on $\mathrm{Lu}_{2} \mathrm{Ir}_{3} \mathrm{Si}_{5}$ [29]. The second and third components differ from $1 / 2$ and $1 / 4$, respectively, by a larger 


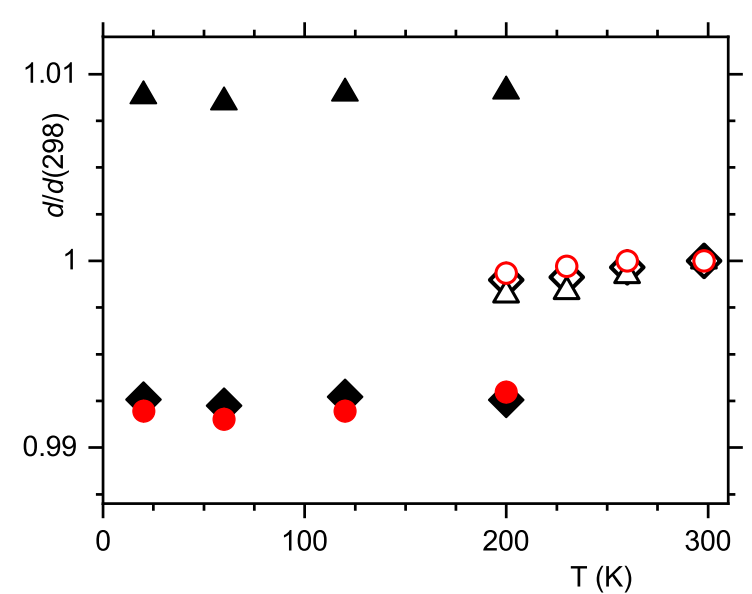

(a)

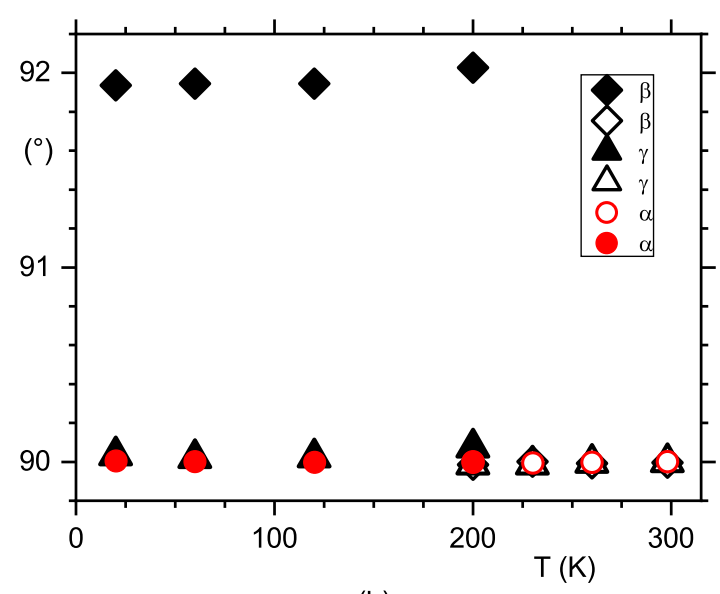

(b)

FIG. 2. Temperature dependence of the lattice parameters. (a) Values of the lattice parameters relative to their values at $T=298 \mathrm{~K}: \frac{a}{a(298)}$ (red circles), $\frac{b}{b(298)}$ (triangles), and $\frac{c}{c(298)}$ (diamonds) with $a(298)=9.9021(4), b(298)=11.3131(5)$, and $c(298)=5.7279(3) \AA$. (b) Values of the lattice parameters $\alpha$ (red circles), $\beta$ (diamonds), and $\gamma$ (triangles). Open symbols are for the orthorhombic phase at $T>200 \mathrm{~K}$; full symbols are for the triclinic phase at $T<200 \mathrm{~K}$. At $200 \mathrm{~K}$ both phases coexist. Two regimes are clearly visible as discontinuities of the temperature dependences of the lattice parameters $a, b, c$, and $\beta$.

amount than for $\mathrm{Er}_{2} \mathrm{Ir}_{3} \mathrm{Si}_{5}$ (Table I). Furthermore, we find that the monoclinic lattice distortion is stronger in $\mathrm{Lu}_{2} \mathrm{Ir}_{3} \mathrm{Si}_{5}[\beta=$ $91.945(2)^{\circ}$ at $\left.60 \mathrm{~K}\right]$ than in $\operatorname{Er}_{2} \mathrm{Ir}_{3} \mathrm{Si}_{5}\left[\beta=91.695(2)^{\circ}\right.$ at $75 \mathrm{~K}]$. The stronger CDW distortion in $\mathrm{Lu}_{2} \mathrm{Ir}_{3} \mathrm{Si}_{5}$ than in $\mathrm{Er}_{2} \mathrm{Ir}_{3} \mathrm{Si}_{5}$ appears to be in agreement with the higher transition temperature in $\mathrm{Lu}_{2} \mathrm{Ir}_{3} \mathrm{Si}_{5}$ (Table I).

The Ibam crystal structures are based on six crystallographically independent atoms, Lu1, Ir1, Ir2, Si1, Si2, and Si3 (Table S8 in the Supplemental Material [35]). The Ir1 atoms form regular zigzag chains along $\mathbf{c}$ with interatomic distance $d[\operatorname{Ir} 1-\operatorname{Ir} 1]=3.657(1) \AA$ at $298 \mathrm{~K}$. For $\mathrm{Er}_{2} \mathrm{Ir}_{3} \mathrm{Si}_{5}$ we have

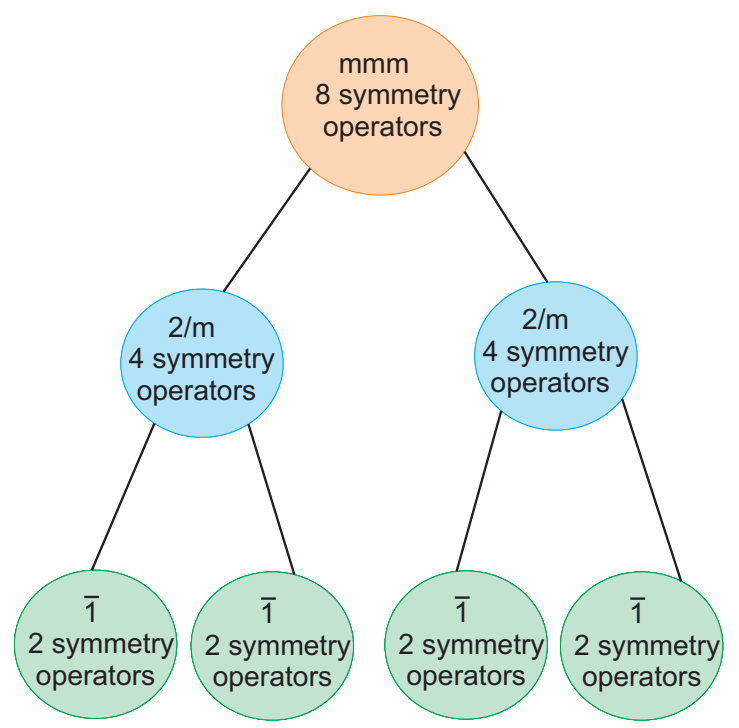

FIG. 3. Flow chart depicting the loss of symmetry from orthorhombic Ibam to triclinic $I \overline{1}$ resulting in the formation of triclinic twin domains of four different orientations. See Nespolo [38] for a formal description of the symmetries of twinned crystals. argued that the Ir1 atoms are responsible for the CDW because the Ir1-Ir1 distance is the most affected by the modulation. Specifically, Ir1 forms (Ir1a, Ir1b) $)_{\infty}$ zigzag chains in the triclinic CDW structure (Fig. 4). The lattice distortion is

TABLE I. Crystal data of the CDW phases of $\mathrm{Lu}_{2} \mathrm{Ir}_{3} \mathrm{Si}_{5}$ (present paper) and $\mathrm{Er}_{2} \mathrm{Ir}_{3} \mathrm{Si}_{5}$ [33].

\begin{tabular}{lcc}
\hline \hline Compound & $\mathrm{Lu}_{2} \mathrm{Ir}_{3} \mathrm{Si}_{5}$ & $\mathrm{Er}_{2} \mathrm{Ir}_{3} \mathrm{Si}_{5}$ \\
\hline$T_{\mathrm{CDW}}{ }^{\mathrm{a}}(\mathrm{K})$ & $202-231$ & $150-166$ \\
$T(\mathrm{~K})$ & 60 & 75 \\
$a(\AA)$ & $9.8182(3)$ & $9.8494(3)$ \\
$b(\AA)$ & $11.4093(3)$ & $11.4863(3)$ \\
$c(\AA)$ & $5.6835(2)$ & $5.7268(2)$ \\
$\alpha\left(^{\circ}\right)$ & $90.001(2)$ & $90.079(1)$ \\
$\beta\left(^{\circ}\right)$ & $91.945(2)$ & $91.695(2)$ \\
$\gamma\left(^{\circ}\right)$ & $90.018(2)$ & $90.051(1)$ \\
$V\left(^{\circ}\right)$ & $636.34(3)$ & $647.60(5)$ \\
$q_{x}$ & $0.2499(3)$ & $0.2495(2)$ \\
$q_{y}$ & $0.4843(4)$ & $0.4973(1)$ \\
$q_{z}$ & $0.2386(2)$ & $0.2483(1)$ \\
Distance Ir1a-Ir1b & & \\
max $(\AA)$ & $3.801(1)$ & $3.818(2)$ \\
min $(\AA)$ & $3.711(1)$ & $3.714(2)$ \\
avg $(\AA)$ & $3.755(1)$ & $3.764(2)$ \\
Distance Ir1a-Ir1b & \\
max $(\AA)$ & & $3.782(2)$ \\
$\min (\AA)$ & $3.761(1)$ & $3.008(2)$ \\
avg $(\AA)$ & $3.002(1)$ & $3.398(2)$ \\
\hline \hline
\end{tabular}

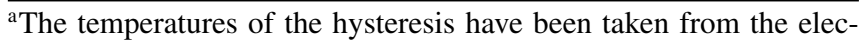
trical resistivity along c, reported in Ref. [30] for $\mathrm{Lu}_{2} \mathrm{Ir}_{3} \mathrm{Si}_{5}$ and in Ref. [33] for $\mathrm{Er}_{2} \mathrm{Ir}_{3} \mathrm{Si}_{5}$.

${ }^{\mathrm{b}}$ Symmetry code for $\operatorname{Ir} 1 \mathrm{~b}(x, y, z)$; given are the maximum (max), minimum ( $\mathrm{min}$ ), and average (avg) distances.

${ }^{\mathrm{c}}$ Symmetry code for $\operatorname{Ir} 1 \mathrm{~b}(x, y, z-1)$. 


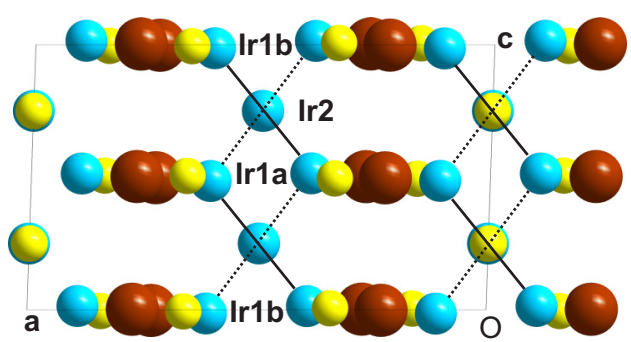

(a)

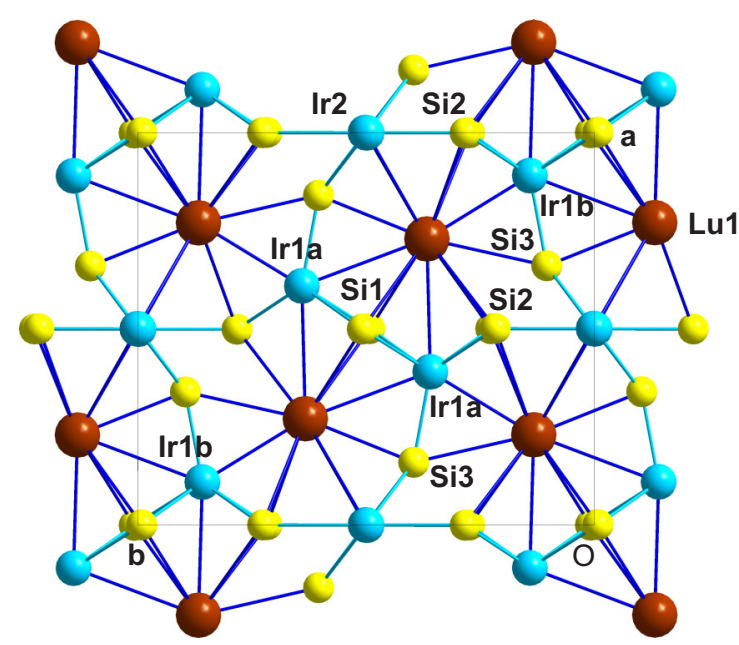

(b)

FIG. 4. Projections of the crystal structure of $\mathrm{Lu}_{2} \mathrm{Ir}_{3} \mathrm{Si}_{5}$ at $60 \mathrm{~K}$. (a) Projection of one unit cell onto the (a, c) plane. Ir1a-Ir1b atoms are at a basic-structure distance of 3.376 (5) $\AA$ for full lines and of 3.753 (5) $\AA$ for dashed lines. (b) Projection of one slab (half a unit cell along c) onto the $(\mathbf{a}, \mathbf{b})$ plane. Atoms Lu1, Ir1, and Si3 are on the $z=0.5$ plane; atoms Si1, Si2, and Ir2 are at $z=0.5 \pm \frac{1}{4}$. Zigzag chains $(\operatorname{Ir} 1 \mathrm{a}, \mathrm{Ir} 1 \mathrm{~b})_{\infty}$ run along $\mathbf{c}$ with the displacement parallel to $\mathbf{a}$.

responsible for alternating short and long Ir1a-Ir1b distances, whereas the shorter distance is the most affected by the modulation. The result is a zigzag chain with a quasi-irregular arrangement of (Ira,Irb) dimers and isolated atoms. For $\mathrm{Lu}_{2} \mathrm{Ir}_{3} \mathrm{Si}_{5}$ we find an identical pattern of modulations (Fig. 5) with interatomic Ir1a-Ir1b distances differing from those in $\mathrm{Er}_{2} \mathrm{Ir}_{3} \mathrm{Si}_{5}$ by less than $0.02 \AA$ (Table I). The unit-cell volume is slightly smaller for $\mathrm{Lu}_{2} \mathrm{Ir}_{3} \mathrm{Si}_{5}$ than for $\mathrm{Er}_{2} \mathrm{Ir}_{3} \mathrm{Si}_{5}$. This is in agreement with the slightly smaller atomic radius of $\mathrm{Lu}$ than of $\operatorname{Er}(2.17$ vs $2.26 \AA)$ [39]. $\mathrm{Lu}_{2} \mathrm{Ir}_{3} \mathrm{Si}_{5}$ is diamagnetic [30], whereas $\mathrm{Er}_{2} \mathrm{Ir}_{3} \mathrm{Si}_{5}$ is paramagnetic with a magnetic moment per $\mathrm{Er}$ atom that is about $10 \%$ lower than the theoretical moment of $\mathrm{Er}^{3+}$ [33]. This major difference is not reflected in the CDW distortions. It might be understood on the basis of our finding that the CDW is supported by $(\operatorname{Ir} 1 \mathrm{a}, \operatorname{Ir} 1 \mathrm{~b})_{\infty}$ zigzag chains, i.e., does not involve the rare-earth atoms.

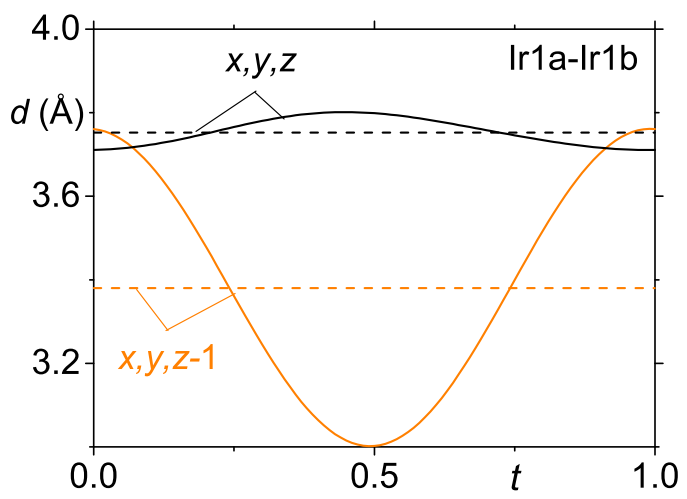

FIG. 5. $t$ plot of the interatomic distances between atoms Ir1a and $\operatorname{Ir} 1 \mathrm{~b}(x, y, z)$ and between $\operatorname{Ir} 1 \mathrm{a}$ and $\operatorname{Ir} 1 \mathrm{~b}$ at $(x, y, z-1)$ for the crystal structure at $T=60 \mathrm{~K}$. Dashed lines give the distances in the basic structure with values of 3.376 (5) and 3.753 (5) $\AA$.
$R_{5} \mathrm{Ir}_{4} \mathrm{Si}_{10}$ is another series of rare-earth $(R) \mathrm{CDW}$ compounds [5], that is built from exactly the same elements as the $R_{2} \mathrm{Ir}_{3} \mathrm{Si}_{5}$ compounds. The change in entropy at the CDW phase transitions is similar for both series of compounds, and with $\Delta S=0.42 R$ for $\mathrm{Lu}_{2} \mathrm{Ir}_{3} \mathrm{Si}_{5}$ [30] it is 5-50× larger than for typical 1D CDW compounds [5]. Properties of $R_{5} \mathrm{Ir}_{4} \mathrm{Si}_{10}$ have been found to depend systematically on $R$. Specifically, $T_{\mathrm{CDW}}$ increases with increasing atomic radius [40]. In contrast, $T_{\mathrm{CDW}}$ is higher for $\mathrm{Lu}_{2} \mathrm{Ir}_{3} \mathrm{Si}_{5}$ than for $\mathrm{Er}_{2} \mathrm{Ir}_{3} \mathrm{Si}_{5}$, despite the smaller atomic radius of $\mathrm{Lu}$. Other differences between the two series include the observations that the CDW is commensurate in $\mathrm{Lu}_{5} \mathrm{Ir}_{4} \mathrm{Si}_{10}$ [41], and it is incommensurate in $\mathrm{Er}_{5} \mathrm{Ir}_{4} \mathrm{Si}_{10}$, whereas the present paper reveals the detailed similarity between CDW distortions in $\mathrm{Lu}_{2} \mathrm{Ir}_{3} \mathrm{Si}_{5}$ and $\mathrm{Er}_{2} \mathrm{Ir}_{3} \mathrm{Si}_{5}$. Furthermore, a lattice distortion (twofold supercell) accompanies the incommensurate CDW in $\mathrm{Er}_{5} \mathrm{Ir}_{4} \mathrm{Si}_{10}$, whereas an additional lattice distortion is not present in $\mathrm{Lu}_{5} \mathrm{Ir}_{4} \mathrm{Si}_{10}$. These differences between the CDWs in the two series support the conclusion that the underlying mechanism is different for the formation of CDWs in the $R_{5} \mathrm{Ir}_{4} \mathrm{Si}_{10}$ and $R_{2} \mathrm{Ir}_{3} \mathrm{Si}_{5}$ systems. In view of the present results one might, thus, conclude that the relevant contributions to the electronic states near the Fermi level are not restricted to Ir atoms in the $R_{5} \mathrm{Ir}_{4} \mathrm{Si}_{10}$ compounds. This is agreement with density functional theory calculations on $\mathrm{Lu}_{5} \mathrm{Ir}_{4} \mathrm{Si}_{10}$ by Mansart et al. [42], who reported involvement of both Ir and Lu $5 d$ orbitals in CDW formation in $\mathrm{Lu}_{5} \mathrm{Ir}_{4} \mathrm{Si}_{10}$.

\section{CONCLUSIONS}

Detailed structural information is provided for the normal and CDW phases of $\mathrm{Lu}_{2} \mathrm{Ir}_{3} \mathrm{Si}_{5}$. The CDW phase is characterized by an incommensurate CDW modulation of the crystal structure, accompanied by a strong lattice distortion achieved through a lowering of the symmetry from orthorhombic to triclinic. The CDW resides on zigzag chains of iridium atoms 
along the $\mathbf{c}$ axis. The structural distortions are completely similar between $\mathrm{Lu}_{2} \mathrm{Ir}_{3} \mathrm{Si}_{5}$ and isostructural $\mathrm{Er}_{2} \mathrm{Ir}_{3} \mathrm{Si}_{5}$ with the small differences explained by the different values of the atomic radii of $\mathrm{Lu}$ and Er. Major differences between $\mathrm{Er}_{2} \mathrm{Ir}_{3} \mathrm{Si}_{5}$ and $\mathrm{Lu}_{2} \mathrm{Ir}_{3} \mathrm{Si}_{5}$ are the higher transition temperature $T_{\mathrm{CDW}}$ and the larger hysteresis of the first-order phase transition of the latter compound. The absence of an effect of the rare-earth element on the CDW distortions is explained by a CDW supported by Ir atom zigzag chains, not involving the rare-earth atoms. We argue that the mechanism of CDW formation is different for $R_{2} \mathrm{Ir}_{3} \mathrm{Si}_{5}$ than for the rare-earth CDW compounds $R_{5} \mathrm{Ir}_{4} \mathrm{Si}_{10}$.

\section{ACKNOWLEDGMENTS}

Single-crystal $\mathrm{x}$-ray diffraction data were collected at Beamline P24 of PETRA-III at DESY, Hamburg, Germany. J.-K.B. acknowledges financial support from the Alexandervon-Humboldt Foundation.
[1] P. Monceau, Electronic crystals: An experimental overview, Adv. Phys. 61, 325 (2012).

[2] M. Roman, J. Strychalska-Nowak, T. Klimczuk, and K. K. Kolincio, Extended phase diagram of $R \mathrm{NiC}_{2}$ family: Linear scaling of the Peierls temperature, Phys. Rev. B 97, 041103(R) (2018).

[3] M. Dressel and S. Tomic, Molecular quantum materials: Electronic phases and charge dynamics in two-dimensional organic solids, Adv. Phys. 69, 1 (2020).

[4] D. I. Khomskii and S. V. Streltsov, Orbital effects in solids: Basics, recent progress, and opportunities, Chem. Rev. 121, 2992 (2021).

[5] S. Ramakrishnan and S. van Smaalen, Unusual ground states in $R_{5} T_{4} X_{10}(R=$ rare earth; $T=\mathrm{Rh}, \mathrm{Ir}$; and $X=\mathrm{Si}, \mathrm{Ge}, \mathrm{Sn}): \mathrm{A}$ review, Rep. Prog. Phys. 80, 116501 (2017).

[6] G. Grüner, Charge Density Waves in Solids (Addison-Wesley, Reading, MA, 1994).

[7] J. C. Marmeggi, A. Delapalme, G. H. Lander, C. Vettier, and N. Lehner, Atomic displacements in the incommensurable chargedensity wave in alpha-uranium, Solid State Commun. 43, 577 (1982).

[8] R. M. Fleming, F. J. DiSalvo, R. J. Cava, and J. V. Waszczak, Observation of charge-density waves in the cubic spinel structure $\mathrm{CuV}_{2} \mathrm{~S}_{4}$, Phys. Rev. B 24, 2850 (1981).

[9] S. Kawaguchi, Y. Kubota, N. Tsuji, J. Kim, K. Kato, M. Takata, and H. Ishibashi, Structural analysis of spinel compound $\mathrm{CuV}_{2} \mathrm{~S}_{4}$ with incommensurate charge-density wave, J. Phys.: Conf. Ser. 391, 012095 (2012).

[10] H. Okada, K. Koyama, and K. Watanabe, Two-step structural modulations and Fermi liquid state in spinel compound $\mathrm{CuV}_{2} \mathrm{~S}_{4}$, J. Phys. Soc. Jpn. 73, 3227 (2004).

[11] S. Ramakrishnan, A. Schönleber, C. B. Hübschle, C. Eisele, A. M. Schaller, T. Rekis, N. H. A. Bui, F. Feulner, S. van Smaalen, B. Bag, S. Ramakrishnan, M. Tolkiehn, and C. Paulmann, Charge density wave and lock-in transitions of $\mathrm{CuV}_{2} \mathrm{~S}_{4}$, Phys. Rev. B 99, 195140 (2019).

[12] A. Ślebarski and J. Goraus, Electronic structure and crystallographic properties of skutterudite-related $\mathrm{Ce}_{3} M_{4} \mathrm{Sn}_{13}$ and $\mathrm{La}_{3} M_{4} \mathrm{Sn}_{13}(M=\mathrm{Co}, \mathrm{Ru}$, and Rh), Phys. Rev. B 88, 155122 (2013).

[13] Y. Otomo, K. Iwasa, K. Suyama, K. Tomiyasu, H. Sagayama, R. Sagayama, H. Nakao, R. Kumai, and Y. Murakami, Chiral crystal-structure transformation of $R_{3} \mathrm{Co}_{4} \mathrm{Sn}_{13}(R=\mathrm{La}$ and $\mathrm{Ce})$, Phys. Rev. B 94, 075109 (2016).

[14] J. Welsch, S. Ramakrishnan, C. Eisele, N. van Well, A. Schönleber, S. van Smaalen, S. Matteppanavar, A.
Thamizhavel, M. Tolkiehn, C. Paulmann, and S. Ramakrishnan, Second-order charge-density-wave transition in single crystals of $\mathrm{La}_{3} \mathrm{Co}_{4} \mathrm{Sn}_{13}$, Phys. Rev. Mater. 3, 125003 (2019).

[15] E. DiMasi, M. C. Aronson, J. F. Mansfield, B. Foran, and S. Lee, Chemical pressure and charge-density waves in rare-earth tritellurides, Phys. Rev. B 52, 14516 (1995).

[16] N. Ru, C. L. Condron, G. Y. Margulis, K. Y. Shin, J. Laverock, S. B. Dugdale, M. F. Toney, and I. R. Fisher, Effect of chemical pressure on the charge density wave transition in rare-earth tritellurides $R \mathrm{Te}_{3}$, Phys. Rev. B 77, 035114 (2008).

[17] A. Banerjee, Y. Feng, D. M. Silevitch, J. Wang, J. C. Lang, H.H. Kuo, I. R. Fisher, and T. F. Rosenbaum, Charge transfer and multiple density waves in the rare earth tellurides, Phys. Rev. B 87, 155131 (2013).

[18] E. DiMasi, B. Foran, M. C. Aronson, and S. Lee, Stability of charge-density waves under continuous variation of band filling in $\operatorname{LaTe}_{2-x} \mathrm{Sb}_{x}(0<x<1)$, Phys. Rev. B. 54, 13587 (1996).

[19] J. H. Shim, J.-S. Kang, and B. I. Min, Electronic Structures of $R \mathrm{Te}_{2}(R=\mathrm{La}, \mathrm{Ce})$ : A Clue to the Pressure-Induced Superconductivity in $\mathrm{CeTe}_{1.82}$, Phys. Rev. Lett. 93, 156406 (2004).

[20] C. N. Kuo, C. J. Hsu, C. W. Tseng, W. T. Chen, S. Y. Lin, W. Z. Liu, Y. K. Kuo, and C. S. Lue, Charge density wave like behavior with magnetic ordering in orthorhombic $\mathrm{Sm}_{2} \mathrm{Ru}_{3} \mathrm{Ge}_{5}$, Phys. Rev. B 101, 155140 (2020).

[21] S. Shimomura, C. Hayashi, G. Asaka, N. Wakabayashi, M. Mizumaki, and H. Onodera, Charge-Density-Wave Destruction and Ferromagnetic Order in $\mathrm{SmNiC}_{2}$, Phys. Rev. Lett. 102, 076404 (2009).

[22] A. Wölfel, L. Li, S. Shimomura, H. Onodera, and S. van Smaalen, Commensurate charge-density wave with frustrated interchain coupling in $\mathrm{SmNiC}_{2}$, Phys. Rev. B 82, 054120 (2010).

[23] S. Shimomura, C. Hayashi, N. Hanasaki, K. Ohnuma, Y. Kobayashi, H. Nakao, M. Mizumaki, and H. Onodera, Multiple charge density wave transitions in the antiferromagnets $R \mathrm{NiC}_{2}$ ( $R=\mathrm{Gd}, \mathrm{Tb})$, Phys. Rev. B 93, 165108 (2016).

[24] K. K. Kolincio, M. Roman, M. J. Winiarski, J. StrychalskaNowak, and T. Klimczuk, Magnetism and charge density waves in $R \mathrm{NiC}_{2}(R=\mathrm{Ce}, \mathrm{Pr}, \mathrm{Nd})$, Phys. Rev. B 95, 235156 (2017).

[25] H. Maeda, R. Kondo, and Y. Nogami, Multiple charge density waves compete in ternary rare-earth nickel carbides, $R \mathrm{NiC}_{2}(R$ : Y, Dy, Ho, and Er), Phys. Rev. B 100, 104107 (2019).

[26] K. K. Kolincio, M. Roman, and T. Klimczuk, Enhanced Mobility and Large Linear Nonsaturating Magnetoresistance in the Magnetically Ordered States of $\mathrm{TmNiC}_{2}$, Phys. Rev. Lett. 125, 176601 (2020). 
[27] Y. Singh, D. Pal, S. Ramakrishnan, A. M. Awasthi, and S. K. Malik, Phase transitions in $\mathrm{Lu}_{2} \mathrm{Ir}_{3} \mathrm{Si}_{5}$, Phys. Rev. B 71, 045109 (2005).

[28] Y. K. Kuo, K. M. Sivakumar, T. H. Su, and C. S. Lue, Phase transitions in $\mathrm{Lu}_{2} \mathrm{Ir}_{3} \mathrm{Si}_{5}$ : An experimental investigation by transport measurements, Phys. Rev. B 74, 045115 (2006).

[29] M. H. Lee, C. H. Chen, M.-W. Chu, C. S. Lue, and Y. K. Kuo, Electronically phase-separated charge-density waves in $\mathrm{Lu}_{2} \mathrm{Ir}_{3} \mathrm{Si}_{5}$, Phys. Rev. B 83, 155121 (2011).

[30] N. S. Sangeetha, A. Thamizhavel, C. V. Tomy, S. Basu, A. M. Awasthi, P. Rajak, S. Bhattacharyya, S. Ramakrishnan, and D. Pal, Multiple charge density wave transitions in singlecrystalline $\mathrm{Lu}_{2} \mathrm{Ir}_{3} \mathrm{Si}_{5}$, Phys. Rev. B 91, 205131 (2015).

[31] R. Padam, A. Thamizhavel, S. Ravi, S. Ramakrishnan, and D. Pal, Solid State Physics: Proceedings of the 56th DAE Solid State Physics Symposium 2011, edited by R. Mittal, A. K. Chauhan, and R. Mikhopadhyay, AIP Conf. Proc. No. 1447 (AIP, New York, 2012), p. 1007.

[32] P. C. Lalngilneia, A. Thamizhavel, S. Ramakrishnan, and D. $\mathrm{Pal}$, Charge density wave in $\mathrm{Er}_{2} \mathrm{Ir}_{3} \mathrm{Si}_{5}$ single crystal, in Solid State Physics: Proceedings of the 58th DAE Solid State Physics Symposium 2013, edited by C. Murli, D. Chattacharyya, and C. S. Gadkari, AIP Conf. Proc. No. 1591, (AIP, New York, 2014), p. 113.

[33] S. Ramakrishnan, A. Schönleber, T. Rekis, N. van Well, L. Noohinejad, S. van Smaalen, M. Tolkiehn, C. Paulmann, B. Bag, A. Thamizhavel, D. Pal, and S. Ramakrishnan, Unusual charge density wave transition and absence of magnetic ordering in $\mathrm{Er}_{2} \mathrm{Ir}_{3} \mathrm{Si}_{5}$, Phys. Rev. B 101, 060101(R) (2020).

[34] W. R. Meier, B. C. Chakoumakos, S. Okamoto, M. A. McGuire, R. P. Hermann, G. D. Samolyuk, S. Gao, Q. Zhang, M. B. Stone, A. D. Christianson, and B. C. Sales, A catastrophic charge density wave in $\mathrm{BaFe}_{2} \mathrm{Al}_{9}$, Chem. Mater. 33, 2855 (2021).

[35] See Supplemental Material at https://link.aps.org/supplemental/ 10.1103/PhysRevB.104.054116 for details on the diffraction experiments, values of the structural parameters at each temperature, and $t$ plots of interatomic distances and displacement modulation, which includes Refs. [43-47].
[36] Agilent Technologies UK, CrysAlisPRO Software (Yarnton, England, 2014).

[37] H. T. Stokes, B. J. Campbell, and S. van Smaalen, Generation of $(3+d)$-dimensional superspace groups for describing the symmetry of modulated crystalline structures, Acta Crystallogr., Sect. A: Found. Crystallogr. 67, 45 (2011).

[38] M. Nespolo, The chromatic symmetry of twins and allotwins, Acta Crystallogr., Sect. A: Found. Adv. 75, 551 (2019).

[39] E. Clementi, D. L. Raimondi, and W. P. Reinhardt, Atomic screening constants from SCF functions. II. Atoms with 37 to 86 electrons, J. Chem. Phys. 47, 1300 (1967).

[40] Y. K. Kuo, F. H. Hsu, H. H. Li, H. L. Huang, C. W. Huang, C. S. Lue, and H. D. Yang, Ionic size and atomic disorder effects on the charge-density-wave transitions in $R_{5} \operatorname{Ir}_{4} \operatorname{Si}_{10}(R=\mathrm{Dy}-\mathrm{Lu})$, Phys. Rev. B 67, 195101 (2003).

[41] B. Becker, N. G. Patil, S. Ramakrishnan, A. A. Menovsky, G. J. Nieuwenhuys, J. A. Mydosh, M. Kohgi, and K. Iwasa, Strongly coupled charge-density wave transition in singlecrystal $\mathrm{Lu}_{5} \mathrm{Ir}_{4} \mathrm{Si}_{10}$, Phys. Rev. B 59, 7266 (1999).

[42] B. Mansart, M. J. G. Cottet, T. J. Penfold, S. B. Dugdale, R. Tediosi, M. Chergui, and F. Carbone, Evidence for a Peierls phase-transition in a three-dimensional multiple chargedensity waves solid, Proc. Natl. Acad. Sci. USA 109, 5603 (2012).

[43] V. Petricek, M. Dusek, and L. Palatinus, Crystallographic computing system JANA2006: general features, Z. Kristallogr.Cryst. Mater. 229, 345 (2014).

[44] V. Dyadkin, P. Pattison, V. Dmitriev, and D. Chernyshov, A new multipurpose diffractometer PILATUS@SNBL, J. Synchrotron Radiat. 23, 825 (2016).

[45] A. M. M. Schreurs, X. Xian, and L. M. J. Kroon-Batenburg, EVAL15: A diffraction data integration method based on $a b$ initio predicted profiles, J. Appl. Crystallogr. 43, 70 (2010).

[46] A. J. M. Duisenberg, L. M. J. Kroon-Batenburg, and A. M. M. Schreurs, An intensity evaluation method: EVAL-14, J. Appl. Crystallogr. 36, 220 (2003).

[47] G. M. Sheldrick, SADABS SOFTWARE, Version 2008/1 (Göttingen: University of Göttingen, 2008). 